\title{
EFICIENCIA Y EFECTIVIDAD DEL SERVICIO DE CATALOGO COLECTIVO DE PUBLICACIONES SERIADAS
}

\author{
B. C. Lazo Rodríguez", M. C. González Martínez" y S. Díaz del Campo *
}

Resumen: Se realiza un estudio de la eficiencia y efectividad del servicio de catálogo colectivo de publicaciones seriadas del Sistema Nacional de Información de Ciencias Médicas de Cuba, en el que se consideran los indicadores costo-tiempo-productividad para medir la eficiencia. La efectividad se evalúa mediante el análisis de la necesidad. Los resultados obtenidos muestran valores positivos en cuanto a la eficiencia, aunque en relación con la efectividad no son tan satisfactorios. Se ofrecen los resultados en forma de números absolutos y en valores porcentuales, que se representan en gráficos estadísticos y tablas.

Palabras clave: Eficiencia, efectividad, catálogo colectivo publicaciones seriadas-Cuba, servicios de información.

\begin{abstract}
The service offered by the union catalog of serial publications that belongs to the collection of the National System for Medical Information of Cuba (Sistema Nacional de Información de Ciencias Médicas de Cuba) was studied. The efficiency and effectiveness of the service was measured through cost-time-productivity indicators. Effectivity was evaluated by necessity analysis. The results for efficiency showed positive values, but were not as satisfactory for the effectiveness values. Results are expressed in graphs and tables as absolute numbers and percentages.

Keywords: Union catalogs of serial publications-Cuba, efficiency, effectiveness, information services.
\end{abstract}

\section{Introducción}

El Catálogo Colectivo de publicaciones seriadas (CC) del Sistema Nacional de Información de Ciencias Médicas (SNICM), surgió en 1969 con el propósito de controlar el fondo de publicaciones de la Red, para dar respuesta a las demandas de usuarios individuales o de instituciones. Este servicio se incorporó como valor añadido al servicio de búsquedas bibliográficas, donde se procesan en su totalidad las referencias obtenidas. La Red Nacional de Información de Ciencias Médicas está compuesta por la Biblioteca Médica Nacional, 44 bibliotecas ubicadas en Centros de Investigación, 150 en hospitales y 161 en policlínicos, por lo que este servicio constituye una de las opciones fundamentales que brinda a los usuarios el Departamento de Servicios de Información (1).

En sus inicios se ofreció de forma manual. Sin embargo, con la explotación de bases de datos bibliográficas en CD-ROM, aumentó considerablemente la demanda de localización de la fuente primaria, por lo que se decidió su automatización.

Con ésta se obtuvieron logros significativos, entre los que se pueden citar como más relevantes: la rapidez en la actualización de la base de datos; la respuesta inmediata a la demanda de localización; la impresión del CC y la copia en disco de la base a todas las instituciones que posean los requerimientos técnicos necesarios.

* Centro Nacional de Información de Ciencias Médicas. La Habana. Cuba.

Recibido 4-4-94. 
Para verificar si el servicio de $\mathrm{CC}$ ha cumplido el propósito trazado en sus inicios, conocer su nivel actual de operación, identificar el grado de satisfacción de las demandas que se le formulan y determinar las acciones que puedan emprenderse para incrementar su rendimiento en el futuro, se realizó un análisis de su eficiencia y efectividad.

Para medir la eficiencia se tomó como punto de referencia la operación interna del servicio, y se consideraron los indicadores costo, tiempo y productividad como los principales factores que la determinan.

La efectividad se evaluó a través del estudio de la necesidad, vista como la satisfacción del usuario en cuanto a la demanda de sus intereses (2).

Con este trabajo se pretende obtener elementos objetivos que contribuyan a la mejora del servicio y que sirvan de base para estudios posteriores.

\section{Material y método}

Para el análisis de la eficiencia económica de CC se relacionaron los indicadores utilizados y se definieron aquellos cuya interpretación así lo hizo necesario.

Análsis del costo:

FTPP: Producto del número de horas a trabajar según el plan en un mes por el total de meses laborados en el año.

FTPR: Sumatoria del número de horas realmente trabajadas en cada mes durante el año, las cuales fueron obtenidas de las estadísticas mensuales de CC para el año 1991.

FTPNA: Diferencia entre el FTPP y el FTPR.

SMP: Cociente del total del salario planificado para los trabajadores de este Frente entre el número de trabajadores.

SMR: Cociente del total del salario real recibido por los trabajadores de CC (descontado el salario devengado por concepto de estudio de acuerdo con el Decreto-Ley 91) entre el número de trabajadores.

$S D$ : Total del salario real recibido por los trabajadores incluyendo el salario por concepto de estudio.

Número de citas a localizar según el plan (citas planificadas): Cantidad de citas que el sistema puede localizar en un tiempo determinado. En el sistema automatizado se consideró 100 citas por hora.

Número de citas localizadas realmente: Cantidad de citas localizadas en correspondencia con el tiempo trabajado.

Número de citas trabajadas: Demanda social de citas a localizar.

PP: Cociente del número de citas a localizar según el plan entre el número de trabajadores.

$P R$ : Cociente del número de citas trabajadas entre el número de trabajadores.

Correlación salario medio-productividad: SMR/SMP/PR/PP.

Gasto en consumo energético de CC: Suma de los gastos en consumo energético de una microcomputadora y su impresora, más el gasto en iluminación por el FTPR, todo ello multiplicado por la tarifa para el sector estatal.

Gasto en insumos de CC: Consumo de todos los materiales utilizados. 
Costo total estimado de CC: Suma de los gastos en insumos, los gastos por consumo energético y el total de salario devengado en 1991.

Costo total de la actividad del CNICM: Total de gastos en que incurrió el centro durante 1991.

Para el estudio del tiempo empleado en la localización de citas se consideraron tres formas de realización: Manual, Automatizado 1, consistente en un sistema compilado en clipper, que para localizar un título requiere la obtención inicial del código que lo representa en la base de datos, y Automatizado 2, representado por un programa en clipper que interactúa automáticamente entre la base de datos creada a partir del primer sistema y la salida de búsqueda bibliográfica de la $\mathrm{BD}$ Medline, para insertar en ésta la ubicación físifca de los documentos. La identificación del título se efectúa comparando el número ISSN; el título abreviado; el Journal code y confrontando posteriormente los campos año, volumen y número.

Para el estudio del procesamiento manual se consideró el periodo comprendido entre enero de 1988 y febrero de 1989; el procesamiento automatizado 1 se utilizó entre marzo de 1989 y febrero de 1992; y para el procedimiento automatizado 2 se tuvo en cuenta el período comprendido entre marzo y julio de 1992.

El análisis del tiempo empleado en el procesamiento reposó en dos indicadores:

$E P E$ : Estadía promedio estandarizada (en días) de las bibliografias localizadas.

$T M P$ : Tiempo medio (en minutos) de procesamiento de una cita.

$E P E$ : Indicador establecido a los efectos del presente estudio. Se define para un día laboral dado como

$$
\mathrm{EP}=\frac{\mathrm{EP}}{\mathrm{C}} \times 100
$$

donde

EP: Estadia promedio (en días) de las bibliografías.

Suma de las estadias (en días) de cada bibliografia cuya fecha de entrada

$\mathrm{EP}=\frac{\text { al } \mathrm{CC} \text { es el día analizado }}{\text { Número de bibliografias recibidas el día analizado }}$

C: Promedio de citas por bibliografia.

$$
\mathrm{C}=\frac{\text { Total de citas en las bibliografias solicitadas en el día analizado }}{\text { Número de bibliografias recibidas el día analizado }}
$$

Para establecer la comparación el indicador EPE asume, mediante la estandarización, que cada bibliografía cuente con igual número de citas, o sea 100.

La estandarización utilizada permite que pueda compararse la estadía de las bibliografias aun cuando difieran sustancialmente en el número de citas que deben localizarse.

Para la obtención de EPE se seleccionaron al azar 10 dias en los que se utilizó el procesamiento automatizado 1 , y otros 10 en los que se empleó el procedimiento automatizado 2. De esta manera se analizaron 139 bibliografias procesadas por el 
primer método y 106 por el segundo. No toda la información necesaria estuvo disponible para el período en que se utilizó el procesamiento manual.

La selección aleatoria de los días a estudiar se realizó teniendo en cuenta que se incluyeran en la muestra todos los días laborables. Se entendió adicionalmente que marzo y mayo eran dos meses en los cuales existe un trabajo estable no afectado por vacaciones de los trabajadores, receso escolar u otros factores exógenos. Colateralmente, con vistas a la comparabilidad de los resultados, para ambos procedimientos de análisis se estudiaron los citados meses.

Los días seleccionados fueron el 1, 4, 12, 20 y 28 de marzo de 1991; el 2, 9, 17, 20 y 28 de mayo de 1991; el 5, 9, 17, 25 y 26 de marzo de 1992; y el 4, 5, 11, 20 y 28 de mayo de 1992. Los dos primeros períodos corresponden a la etapa en que se utilizaba el procesamiento automatizado $1, y$ los dos restantes al momento en que se empleaba el procesamiento automatizado 2.

Por otra parte, el indicador TMP, o sea, el tiempo medio de procesamiento de una cita (en minutos) para un mes dado se define como:

donde

$$
\mathrm{TMP}=\frac{\mathrm{h}}{\mathrm{p}} \times 60
$$

h: tiempo total de máquina (en horas) empleadas en procesar las citas.

p: número de citas procesadas en el mes considerado.

Fue de interés obtener este indicador para todos los meses comprendidos entre enero de 1988 y julio de 1992. Sin embargo, no pudo calcularse por falta de uno u otro dato, o ambos, en enero, febrero, marzo, abril y agosto de 1988.

\section{Fuentes de información}

El número de horas planificadas y trabajadas, el total de salario planificado y devengado, así como el número de trabajadores y el gasto en insumos, se obtuvieron del Dpto. Económico del centro.

El número de citas planificadas y trabajadas, la cantidad de bibliografias y el tiempo total de máquina (horas) empleadas en procesar las citas, se obtuvieron del Frente de CC.

Por último, el gasto en consumo energético, iluminación y la tarifa en el sector estatal, se obtuvieron de la Dirección de Inversiones del Ministerio de Salud Pública (MINSAP).

La evaluación de la efectividad del servicio se realizó a través del análisis de la demanda de localización de citas bibliográficas según especialidades médicas.

Para el estudio de la misma se seleccionó una muestra de 290 búsquedas bibliográficas durante el primer trimestre de 1992, se consideraron las especialidades médicas registradas en el Anuario Estadístico del MINSAP 1991. Se confeccionó un modelo (Anexo 1) en el que se registró la demanda de localización. Se estudió la demanda de citas trabajadas según especialidad y se compararon las citas reales y las NO localizadas. Se analizó la relación demanda-respaldo según especialidad.

Se mostraron las causas de las citas NO localizadas y se identificaron los principales títulos de revista según éstas. 
Los resultados obtenidos se resumieron en números absolutos y porcentajes, y se representaron en gráficos estadísticos.

\section{Resultados y discusión}

\section{Análisis de la eficiencia}

Aspecto económico de la eficiencia en la actividad de Catálogo Colectivo durante el año 1991.

Si analizamos la utilización del fondo de tiempo productivo en el año 1991 en el CC observaremos que el FTPP fue de 2.250 horas, mientras que el FTPR, fue de 2.005 horas, para el $89,1 \%$ de aprovechamiento del fondo de tiempo de que se disponia según el plan (ver Anexo 2).

El FTPA fue de 245 horas, que representó el 10,8\% del FTPP (ver Anexo 2).

La correlación salario medio-productividad del trabajo durante el año 1991 resultó de 0,86 , lo que indica que se incrementó la productividad de los trabajadores en relación con el salario devengado por éstos, lo que denota eficiencia económica (ver Anexos 3 y 4).

Los gastos en consumo energético y en insumos fueron de $\$ 234,58$ y $\$ 286,94$ respectivamente, mientras que el total de salario devengado ascendió a $\$ 11.783,80$. Así, el costo total estimado del servicio, fue de \$12.305,54 (ver Anexo 5).

Si tenemos en cuenta que el costo total de la actividad del CNICM en el año 1991, fue de $\$ 1.191 .992,00$ observaremos que el costo de CC representó el 1,03\% de éste.

La EPE registrada en el método automatizado 1 fue de 5,08 días, y con el automatizado 2 fue de 0,41 días, es decir, se redujo en más de 12 veces (figura 1). Se obtuvo una $t=8,31$ con 243 grados de libertad para una $p<0,00001$, o sea, la estadia se redujo muy significativamente al introducirse el método automatizado 2 para el procesamiento.

\section{Figura 1}

\section{Estadía Promedio Estandarizada global de las bibliografías localizadas según tipo de procesamiento}

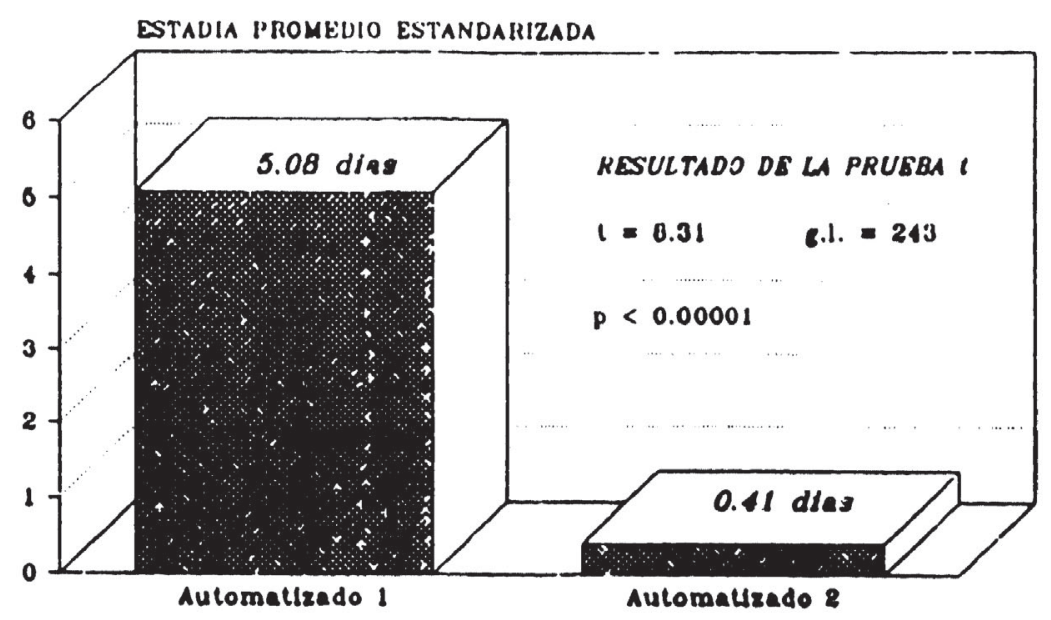


El tiempo medio de procesamiento de una cita se redujo al sustituirse el procesamiento manual por el método automatizado $1 \mathrm{y}$, a su vez, aún más disminuyó al introducirse el método automatizado 2 (figura 2).

Figura 2

Tiempo Medio de Procesamiento de una cita, según método empleado

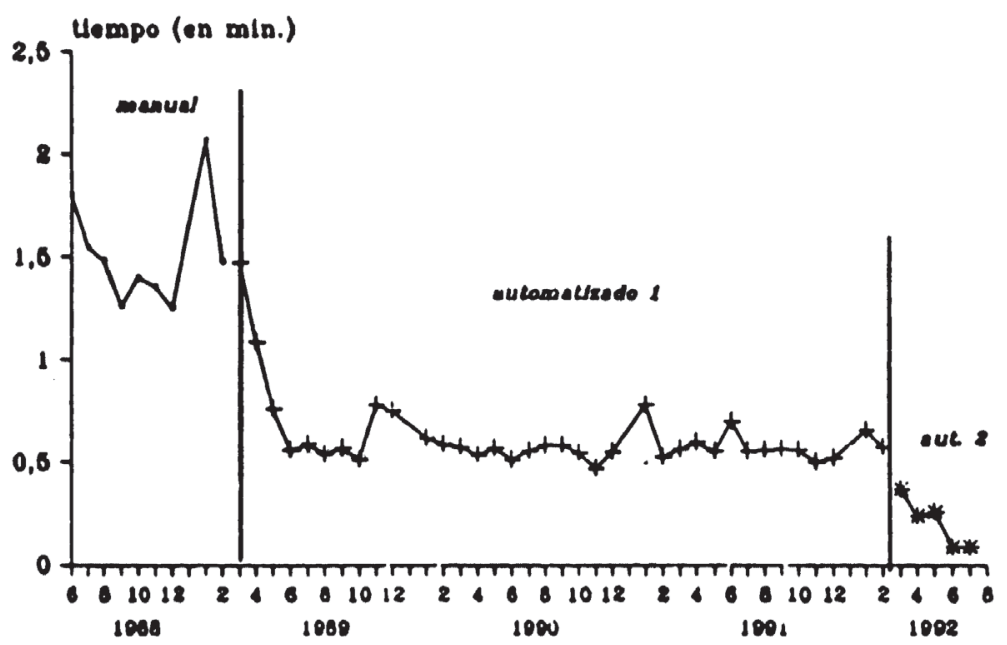

Durante el procesamiento manual el tiempo medio de procesamiento de una cita fue de 1,52 min; disminuyó aproximadamente en dos veces y media al sustituirse este por el automatizado 1 (0,63 min como promedio); y se redujo a sólo 0,23 min por cita al introducirse el procedimiento automatizado 2 .

\section{Análisis de la efectividad}

Se realizaron un total de 290 solicitudes de consultas bibliográficas, correspondientes a 59 especialidades. De éstas, las que más demandaron el servicio (por encima de diez solicitudes) fueron: Medicina General Integral, Pediatría, Medicina Interna, Cirugía General, Ginecología y Obstetricia, Farmacología, Psicología e Inmunología (figura 3).

Figura 3

Principales especialidades según demanda

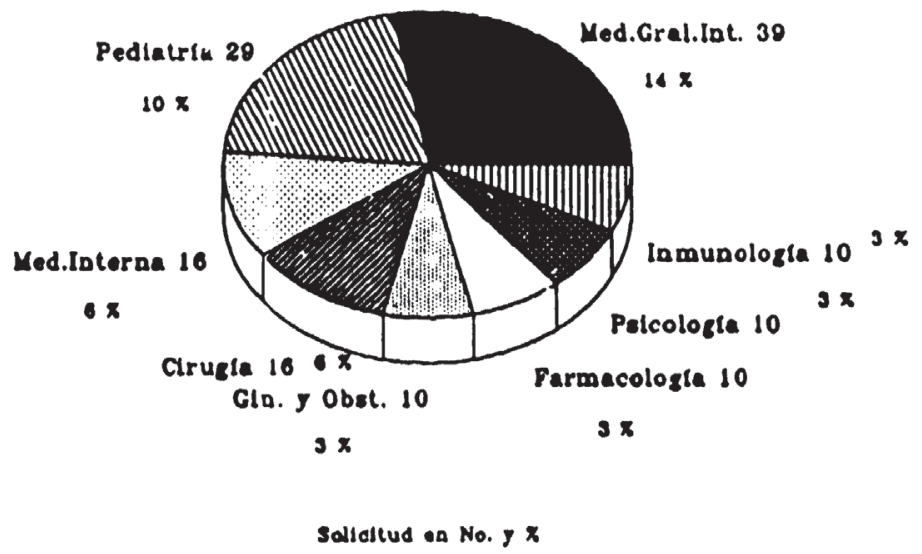


En la figura 4 se observa que de 20.089 citas trabajadas, se encontraron localizados físicamente 5.260 documentos, para un $26,18 \%$ de respaldo que ofrece el CC respecto a la base de datos MEDLINE; en general todas las especialidades registraron un porcentaje inferior al $85 \%$. Las especialidades de ortodoncia, neonatología, nutrición y coloproctología se agrupan por encima del $40 \%$ (figura 5 ).

\section{Figura 4}

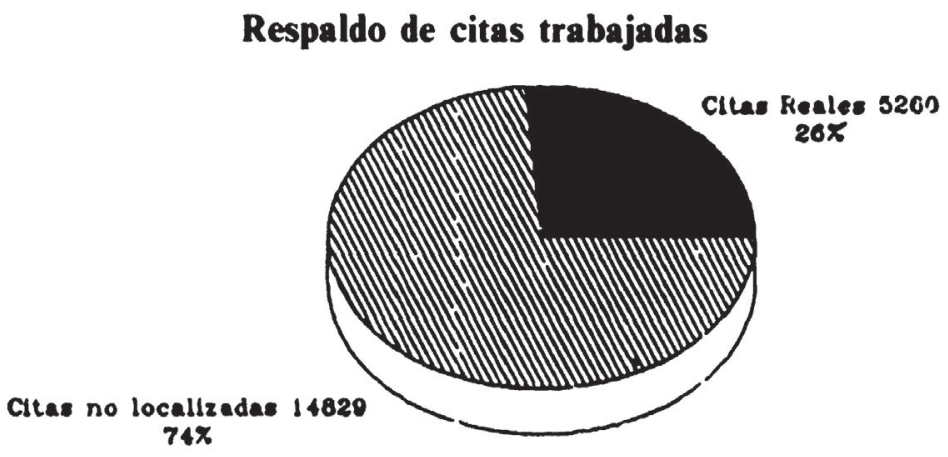

Figura 5

Especialidades de mayor respaldo

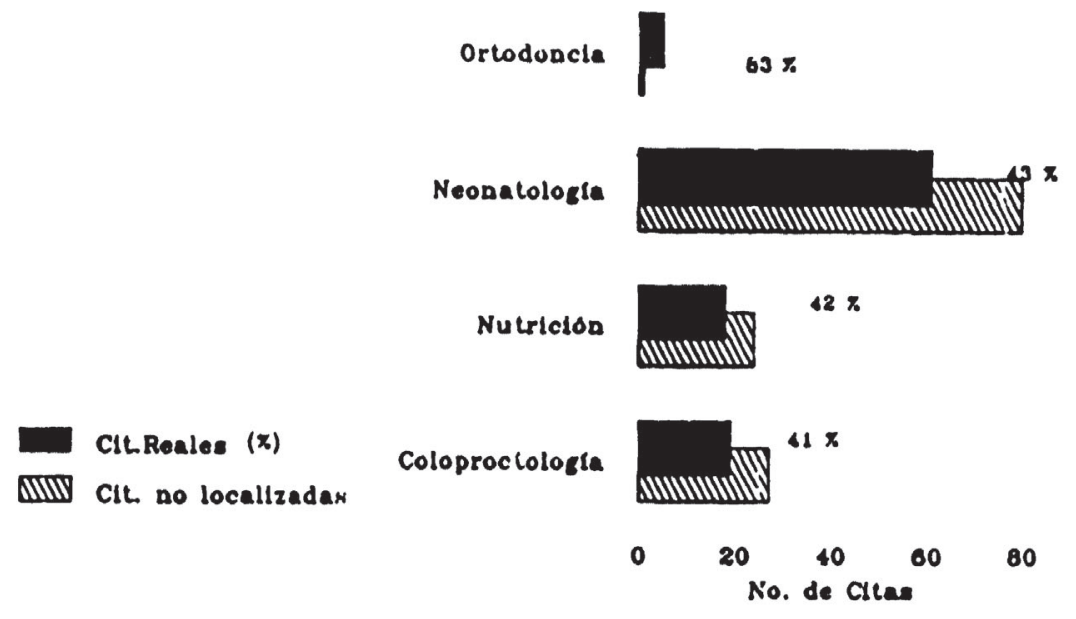

Al analizar la relación demanda-respaldo, observamos que aquellas especialidades registradas entre las de mayor demanda, obtuvieron un respaldo real por debajo del $40 \%$, como es el caso de Medicina General Integral, que sólo obtuvo un $22,17 \%$ (figura 6 ).

El total de citas NO localizadas ascendió a 14.829 (73,82\%). Observamos que existen dos causas fundamentales, dadas por: no existir la revista en el CC $(8.017$ citas) para un $54,06 \%$, o por la no presencia del número en la colección $(6.812$ citas) para un $45,94 \%$.

Se reportaron 1.588 títulos no localizados para una demanda de 7.903 ; resultó el más solicitado la revista Progress in Clinical and Biological Research (84 veces), para un $1,06 \%$ del total. Los 10 títulos representados acumulan aproximadamente el $7,11 \%$ de la demanda total (Tabla $\mathbf{I}$ ). 
Figura 6

Respaldo de citas, según especialidades de mayor demanda

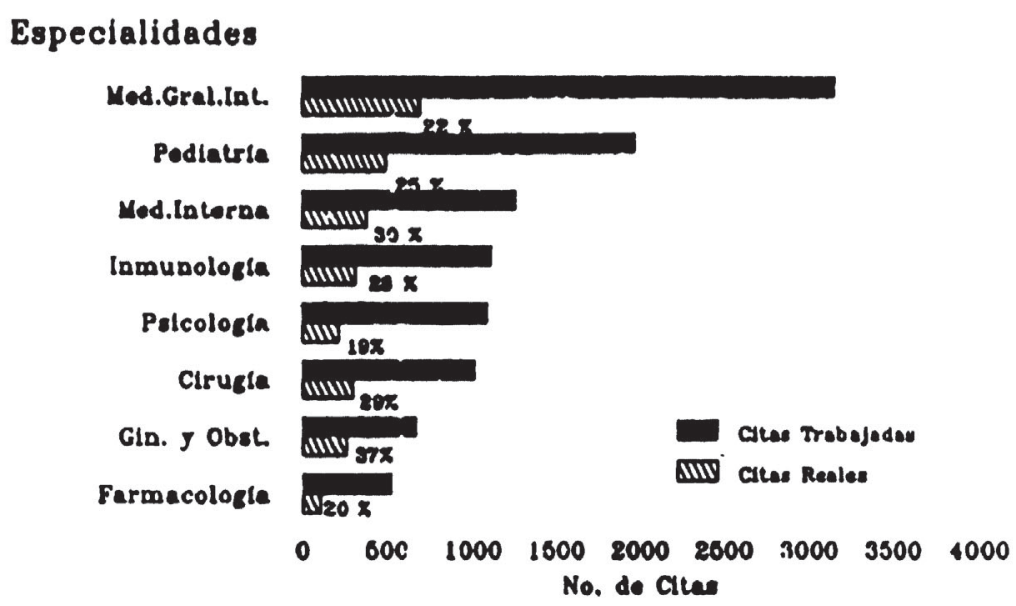

Tabla I

Principales títulos no localizados, según demanda

\begin{tabular}{lccc}
\hline \multicolumn{1}{c}{ Títulos } & Demanda & $\circ$ & ${ }^{\circ}$ "acumulado \\
\hline Prog. Clin. Biol. Res. & 84 & 1,06 & 1,06 \\
J. Tradit. Chin. Med. & 68 & 0,86 & 1,92 \\
Adolescence & 63 & 0,80 & 2,72 \\
J. Adolesc. Health Care & 58 & 0,73 & 3,45 \\
Genitourin Med. & 52 & 0,66 & 4,11 \\
N Z Med. J. & 51 & 0,65 & 4,76 \\
J. Am. Acad. Child Adolesc Psychiatry & 51 & 0,65 & 5,41 \\
Indian Pediatr. & 48 & 0,61 & 6,02 \\
Gastrointest. Endosc. & 44 & 0,56 & 6,58 \\
Ann. NY Acad Sci. & 42 & 0,53 & 7,11 \\
\hline
\end{tabular}

Se reportaron además 882 títulos que presentaban la colección incompleta, para una demanda de 6.926 localizaciones. Entre estos casos se destaca en primer lugar la revista Pediatrics, solicitada 96 veces, que representó un 1,39\% del total. Los 10 títulos representados acumulan aproximadamente el 9,19\% de la demanda (Tabla II).

El análisis de estos datos nos sugiere que es necesario adecuar las adquisiciones, acorde con la base de datos bibliográfica utilizada por nuestra Red de Información (MEDLINE) para garantizar el respaldo con la fuente primaria, de un porcentaje adecuado de la demanda, que se calcula debe ser de un $80 \%$ (3). Por otra parte se deben priorizar las especialidades más demandadas, y lograr completar las colecciones. Para conseguirlo se pueden emprender proyectos de creación de microfichas o la solicitud de reprints a los autores, lo que como es sabido no resulta muy efectivo para este fin, pero crea un fondo de artículos existentes en la institución que contribuye a salvar un tanto esta situación. 
Tabla II

Principales títulos incompletos, según demanda

\begin{tabular}{lccc}
\hline \multicolumn{1}{c}{ Títulos } & Demanda & " & " acumulado \\
\hline Pediatrics & 96 & 1,39 & 1,39 \\
BMJ & 78 & 1,13 & 2.52 \\
South Med. J. & 73 & 1,05 & 3.57 \\
J. Trauma & 63 & 0,91 & 4,48 \\
J. Urol & 61 & 0,88 & 5.36 \\
Br. J. Psychiatry & 56 & 0,81 & 6.17 \\
Am. J. Cardiol. & 54 & 0,78 & 6,95 \\
Am. J. Clin. Nutr. & 54 & 0,78 & 7,73 \\
Soc. Sci. Med. & 54 & 0,78 & 8,51 \\
Ann. Intern. Med. & 47 & 0,68 & 9,19 \\
\hline
\end{tabular}

Es de vital importancia que en el CC estén incluidas todas aquellas instituciones con intereses comunes a nuestro sistema, que posean fondos ampliamente representativos. Además, a la hora de elaborar planes de compras, se debe evitar en lo posible el solapamiento entre las colecciones, lo cual permite ampliar el universo de publicaciones.

Es deber de todas las instituciones que integren el CC permitir el acceso a la información a todos aquellos que la necesiten.

Debemos trazarnos como objetivo el fortalecimiento del CC, para convertirlo en un verdadero registro nacional de ciencias médicas, lo que redundará en un mayor beneficio para el usuario, al obtener una información más completa y efectiva acerca de lo que realmente existe en el país.

\section{Conclusiones}

El análisis de los indicadores costo, tiempo y productividad demostró la eficiencia alcanzada por el servicio, lograda por el bajo costo de la actividad, la rapidez en la respuesta y la alta productividad obtenida.

El estudio de la efectividad demostró que el servicio no satisface todas las necesidades de información de los usuarios, debido al bajo porcentaje de respaldo al documento primario.

\section{Agradecimientos}

Los autores agradecen la asesoría prestada por la Dra. Alina González Borrell y el Prof. Cándido M. López Pardo, así como al resto del colectivo de nuestro frente de trabajo que colaboró en la toma de la muestra.

\section{Bibliografia}

1. VAldes ABReU, M. C., y LAZO RODRIGUEZ, B. C. Catálogo Colectivo de publicaciones seriadas: estudio en el Centro Nacional de Información de Ciencias Médicas. Superación Técnica. 1988 (2), 1-32. 
2. UNESCO. Pautas para la evaluación de sistemas y servicios de información. París: UNESCO, 1984, 1-177.

3. FEDERACION INTERNACIONAL DE ASOCIACIONES DE BIBLIOTECARIOS. Directrices para el establecimiento de catálogos colectivos de publicaciones seriadas. París, UNESCO, 1967, 1-30.

\section{ANEXO 1 \\ Modelo control de la demanda}

No. Planilla

Especialidad

\begin{tabular}{lccc}
\hline Temas & Citas trabajadas & Citas reales & Citas no localizadas \\
\hline No. título & No título (causa .1) & No número (causa 2) \\
\hline 1. & & \\
2. \\
3. \\
4. \\
5. \\
6. \\
Totales \\
\hline
\end{tabular}

\section{ANEXO 2}

Cálculo de la utilización del fondo de tiempo productivo en Catálogo Colectivo en 1991

$$
\begin{aligned}
& \mathrm{FTPP}=\left(\begin{array}{c}
150 \text { horas } \times 12 \text { meses }) \\
\text { Máquina } 1
\end{array}\right)+\left(\begin{array}{c}
37,5 \mathrm{~h} \times 12 \mathrm{~m} \\
\text { Máquina } 2
\end{array}\right)=2.250 \mathrm{~h} \\
& \mathrm{FTPR}=\sum_{\mathrm{m}=1}^{12} \mathrm{E} \quad \mathrm{FTPR}_{\mathrm{m}}=2.005 \mathrm{~h} \\
& \text { FTPNA }=\text { FTPP }- \text { FTPR }=2.250-2.005=245 \mathrm{~h}
\end{aligned}
$$




\section{ANEXO 3}

Salario devengado por los trabajadores del CC durante el ano 1991

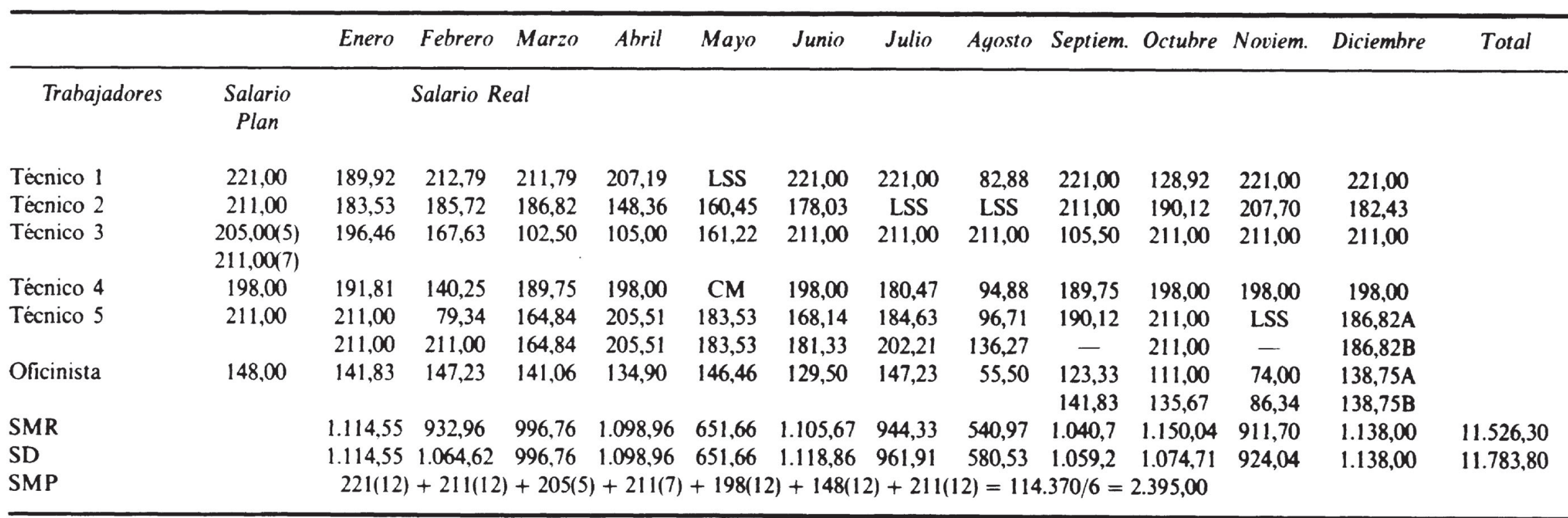

LSS: Licencia sin sueldo.

CM: Certificado Médico.

Decreto 91: Decreto estatal mediante el cual los trabajadores reciben un préstamo para cursar estudios en la enseñanza superior.

A: Salario que corresponde al tiempo trabajado, lo que resta de lo devengado es por Decreto 91.

B: Lo devengado en el mes. 


\section{ANEXO 4}

Cálculo de correlación salario medio-productividad del trabajo en catálogo colectivo en el afio 1991

Dado

$$
\begin{aligned}
& \text { SMP }=\$ 2,395.00 \\
& S M R=\$ 1,921.05
\end{aligned}
$$

Citas a localizar plan $=225.000$ (consideradas 100 en una hora).

Citas localizadas real $=209.716$.

Trabajadores de $\mathrm{CC}=5$ técnicos.

Entonces

$$
\begin{aligned}
& \operatorname{Pp}=\frac{225.000}{5}=45.000 \\
& \operatorname{Pr}=\frac{209.716}{5}=41.943
\end{aligned}
$$

Correlación salario medio/productividad:

$$
\frac{\frac{\text { SMR }}{\text { SMP }}}{\frac{P R}{\text { PP }}}=\frac{\frac{1.921,05}{2.395,00}}{\frac{41.943}{45.000}}=0,86
$$

\section{ANEXO 5}

Cálculo del costo de la actividad de catálogo colectivo en el ańo 1991

Sea

$$
\operatorname{COSTO}=\mathrm{SD}+\mathrm{I}+\mathrm{E}
$$

siendo

SD: Ya definido.

$I$ = Proporción de trabajadores de CC respecto al total de trabajadores $\times$ insumos del departamento.

$\mathrm{E}=\mathrm{FTPR} \times \mathrm{G} \times \mathrm{T}$

donde

FTPR: Ya está definido.

G: Gasto de energía en microcomputadoras e impresoras + gasto en iliminación.

T: Tarifa eléctrica del sector estatal. 
Se tiene que

$\mathrm{SD}=\$ 11.783,80$

Proporción de trabajadores de CC respecto al total de trabajadores $=6 / 40=0,15$.

FTPR $=2.005$

Insumos del Dpto. $=\$ 1.912,91$

$\mathrm{G}=0,3 \mathrm{kw}+1 \mathrm{kw}=1,3 \mathrm{kw}$

$\mathrm{T}=\$ 0,09 \mathrm{kw} / \mathrm{h}$

luego,

$I=0,15 \times \$ 1.912,91=\$ 286,94$

$\mathrm{E}=2.005 \times 1,3 \times \$ 0,09=\$ 234,58$

por tanto

COSTO $=11.783,80+286,94+234,58=12.305,54$ 